\title{
Hiperglicemia y volumen plaquetario medio como predictores tempranos del síndrome coronario agudo tipo IAM SEST
}

\section{Hyperglycemia and mean platelet volume as early predictors of acute coronary syndro- me type IAM SEST}

\author{
Héctor Fabián Ortega Castillo ${ }^{1}$ \\ Juan Carlos Piedra Cosíos ${ }^{2}$
}

${ }^{1}$ Servicio de Medicina Interna, Hospital General IESS, Riobamba, Ecuador.

${ }^{2}$ Servicio de Medicina Interna, Hospital de las Fuerzas Armadas, Quito, Ecuador.

Autor de correspondencia: Héctor F. Ortega, Servicio de Medicina Interna, Hospital General IESS-Riobamba, Avenida Unidad Nacional y Chile, Riobamba, Ecuador; hect_fabian@hotmail.com, 032947481 /0987921749.

\section{RESUMEN}

Introducción: el objetivo del presente trabajo de investigación es determinar la validez diagnóstica que tiene la hiperglucemia y el volumen plaquetario medio en el diagnóstico temprano de pacientes que acuden al Departamento de Emergencia con dolor torácico y sospecha de infarto agudo de miocardio sin elevación del ST. Materiales y métodos: se realizó un ensayo clínico no controlado para validación de pruebas diagnósticas de 6 meses de duración, en 133 pacientes admitidos en el Departamento de Emergencia del Hospital Carlos Andrade Marín por dolor torácico y sospecha de síndrome coronario agudo. Un punto de cohorte de hiperglucemia $>140 \mathrm{mg} / \mathrm{dl}$ y volumen plaquetario medio $\geq 10,33 \mathrm{fl}$ a la admisión fue considerado tomando en cuenta valores propuestos en la curva ROC (curva operante receptor); así como también, se determinó el rendimiento diagnóstico de las pruebas, regresión univariante y multivariante. Resultados: de los 133 pacientes incluidos en el trabajo, 32 (24,1\%) tuvieron infarto agudo de miocardio sin elevación del ST. El rendimiento diagnóstico del volumen plaquetario medio $\geq 10,33 \mathrm{fl}$ (AUC: 0,91; IC 95\%: 0,841-0,979; $<<0,05$ ) tuvo una sensibilidad del $81,2 \%$, especificidad de $93,1 \%$, valor predictivo positivo $78,8 \%$, valor predictivo negativo 94\%, razón de verosimilitud positivo 11,723 y razón de verosimilitud negativo 0,201. Para la hiperglucemia >140 mg/dl (AUC: 0,923; IC 95\%: 0,879-0,967; p<0,05) la sensibilidad fue del $71,9 \%$, especificidad de $90,1 \%$, valor predictivo positivo $69,7 \%$, valor predictivo negativo 91\%, razón de verosimilitud positivo 7,259 y razón de verosimilitud negativo 0,312. Conclusiones: la hiperglucemia y el volumen plaquetario medio son biomarcadores con buena capacidad predictiva para el diagnóstico temprano del infarto agudo de miocardio sin elevación del ST.

Palabras clave: dolor torácico, hiperglucemia, volumen plaquetario medio, síndrome coronario agudo. 


\begin{abstract}
Introduction: the aim of this research is to determine the diagnostic validity of hyperglycemia and mean platelet volume in the early diagnosis of patients who come to the Emergency Department with chest pain and suspected acute myocardial infarction without ST elevation. Materials and methods: an uncontrolled clinical trial was conducted to validate diagnostic tests of 6 months in 133 patients admitted to the Emergency Department of the Carlos Andrade Marín Hospital for chest pain and suspected acute coronary syndrome. A cohort point of hyperglycemia $>140 \mathrm{mg} / \mathrm{dl}$ and mean platelet volume $\geq 10.33 \mathrm{fl}$ upon admission was considered taking into account values proposed in the ROC curve (receiver operant curve); as well as, the diagnostic performance of the tests, univariate and multivariate regression was determined. Results: of the 133 patients included in the study, 32 (24.1\%) had acute myocardial infarction without ST elevation. The diagnostic yield of mean platelet volume $\geq 10.33 \mathrm{fl}$ (AUC: $0.91,95 \%$ CI: $0.841-0.979, \mathrm{p}<0.05$ ) had a sensitivity of $81.2 \%$, specificity of $93.1 \%$, value positive predictive $78.8 \%$, negative predictive value $94 \%$, positive likelihood ratio 11.723 and negative likelihood ratio 0.201. For hyperglycemia $>140 \mathrm{mg} / \mathrm{dL}$ (AUC: 0.923 , $95 \%$ CI: $0.879-0.967, \mathrm{p}<0.05$ ) the sensitivity was $71.9 \%$, specificity $90.1 \%$, positive predictive value $69.7 \%$, negative predictive value $91 \%$, positive likelihood ratio 7,259 and negative likelihood ratio 0,312. Conclusion: hyperglycaemia and mean platelet volume are biomarkers with good predictive capacity for the early diagnosis of acute myocardial infarction without ST elevation.
\end{abstract}

Keywords: thoracic pain, hyperglycemia, medium platelet volume, acute coronary syndrome.

\title{
INTRODUCCIÓN
}

El dolor torácico representa aproximadamente 6 millones de visitas anuales al Departamento de Urgencias en los Estados Unidos. Se calcula que en 2015 murieron por esta causa 17,7 millones de personas, lo cual representa un $31 \%$ de todas las muertes registradas en el mundo. El síndrome coronario agudo corresponde a aproximadamente 12 al 15\% de todas las causas. La enfermedad cardiovascular fue responsable de aproximadamente 8 millones de muertes en el mundo para el año 2013. ${ }^{(1,2)}$

En 2013, las principales causas de muerte en el Ecuador resultaron las enfermedades hipertensivas (tasa de mortalidad de 26,56), cerebrovasculares (tasa de mortalidad de 22,61) y cardiovasculares (tasa de mortalidad de 18,65$).{ }^{(3)}$

A esta realidad se suman factores extrínsecos que propenden de una u otra forma al incremento de estas tasas de mortalidad, entre los que se puede señalar a los servicios de salud saturados, principlamente en el departamento de emergencia de los hospitales de segundo y tercer nivel, por lo que este tipo de patologías se complican debido al retraso en la aplicación de intervenciones y protocolos. 
La necesidad de un diagnóstico temprano en el síndrome coronario agudo es esencial para evitar complicaciones que podrían modificar el pronóstico funcional y vital a corto y mediano plazo en pacientes que acuden por dolor torácico a un servicio de urgencias. Dicha necesidad está directamente relacionada con el beneficio de las diferentes medidas terapéuticas, ya sean éstas técnicas de reperfusión coronaria y/o pautas farmacológicas. ${ }^{(4)}$ Partiendo de este principio elemental, los errores diagnósticos y una alta médica prematura desde el servicio de urgencias constituyen un problema de carácter médico legal importante.

En Ecuador, no se brinda utilidad diagnóstica, ni la prevalencia de la hiperglucemia y el volumen plaquetario medio elevado en pacientes con síndrome coronario agudo, menos aún se utiliza su determinación como un método práctico, rápido y de bajo costo para realizar un adecuado tamizaje de pacientes con dolor torácico en la unidad de urgencias de los hospitales locales. Por tal razón, planteamos la realización del presente trabajo de investigación, cuyo objetivo es establecer el rol de estos marcadores bioquímicos para mejorar el diagnóstico temprano de pacientes con síndrome coronario agudo tipo infarto de miocardio sin elevación del ST.

\section{MATERIAL Y MÉTODOS}

Se llevó a cabo un estudio observacional analítico para validación de pruebas diagnósticas. Utilizando la base de datos disponible en el sistema informático AS400, se procedió a verificar de forma retrospectiva el universo de pacientes que acudieron al Servicio de Emergencia del Hospital Carlos Andrade Marín con dolor torácico y sospecha de síndrome coronario agudo durante el período enero a junio del 2013, utilizando la técnica de muestreo aleatorio simple se seleccionaron 304 pacientes $(\mathrm{N}=1456)$, de los cuales fueron excluidos 171 por presentar los siguientes criterios: edad menor de 18 años, trauma de tórax como causa del dolor torácico, falla renal y hepática previa, trastornos neurológicos agudos, sepsis de cualquier origen, electrocardiograma compatible con IAM con elevación del ST, alta probabilidad clínica de TEP, arritmia cardíaca, consumo de terapia anticoagulante oral, desórdenes mieloproliferativos y malignidad previa, diabetes mellitus tipo 1 y 2 , más de 4 horas de evolución del cuadro clínico.

La población seleccionada (133) debía disponer en su evaluación inicial (admisión en el departamento de emergencia) niveles de glicemia, volumen plaquetario medio, determinación de troponina ultrasensible y estudio electrocardiográfico de doce derivaciones. 
Las variables a ser analizadas fueron: la edad, sexo, niveles de glicemia (mg/dl), volumen plaquetario medio (fl), puntaje de riesgo TIMI, hallazgos electrocardiográficos, características del dolor torácico. El diagnóstico de infarto agudo de miocardio se estableció siguiendo las guías de the European Society of Cardiology/American College of Cardiology, basándose en la positividad de biomarcadores cardíacos (troponina ultrasensible) con un valor superior al percentil 99 del límite superior normal, asociado con los siguientes parámetros: síntomas de isquemia (dolor torácico que cumple los siguientes criterios: 1.- tipo opresivo de intensidad variable localizado en región precordial, con irradiación a cuello, hombro y brazo izquierdo; acompañado de síntomas neurovegetativos, 2.- aparece en actividad, y 3.- alivia con el reposo y/o nitratos), hallazgos electrocardiográficos (depresión del segmento ST de al menos $0,05 \mathrm{mv}$ en 2 o más derivaciones contiguas, inversión de la onda $\mathrm{T}$ de al menos 0,1 $\mathrm{mv}$, onda $\mathrm{Q}>30 \mathrm{~ms}$ en ancho y mayor o igual a $0,1 \mathrm{mv}$ en profundidad en dos derivaciones contiguas, cambios nuevos en la onda $\mathrm{T}$ o segmento $\mathrm{ST}$, nuevo bloqueo completo de rama izquierda), evidencia en imagen de alteración en la viabilidad miocárdica o pérdida segmentaria de la contractilidad.

Se consideró positividad en las variables a correlacionar si cumplían con los siguientes criterios:

\section{Dolor torácico}

POSITIVO: cumple 2 o 3 de los siguientes criterios:

- Tipo opresivo de intensidad variable localizado en región precordial, con irradiación a cuello, hombro y brazo izquierdo; acompañado de síntomas neurovegetativos.

- Apareción en actividad.

- Alivio con el reposo y/o nitratos.

NEGATIVO: cumple 1 criterio

\section{Electrocardiograma}

POSITIVO:

- Depresión del segmento ST de al menos 0,05 mv en 2 o más derivaciones contiguas.

- Inversión de la onda T de al menos 0,1 mv.

- Onda $\mathrm{Q}>30 \mathrm{~ms}$ en ancho y mayor o igual a $0,1 \mathrm{mv}$ en profundidad en dos derivaciones contiguas.

NEGATIVO: trazado electrocardiográfico NORMAL (no cambios isquémicos preexistentes).

\section{Puntaje TIMI NSTEMI}

POSITIVO: puntuación en 1 o más parámetros $(\geq 1)$.

NEGATIVO: ausencia de algún parámetro a calificar $(0)$. 


\section{Niveles de troponina ultrasensible (ng/L)}

POSITIVO: $\geq 14 \mathrm{ng} / \mathrm{L}$

NEGATIVO: $<14 \mathrm{ng} / \mathrm{L}$

\section{Hiperglucemia}

POSITIVO: > $140 \mathrm{mg} / \mathrm{dl}$

NEGATIVO: $\leq 140 \mathrm{mg} / \mathrm{dl}$

\section{Volumen Plaquetario Medio (VPM)}

POSITIVO: $\geq 10,33 \mathrm{fl}$

NEGATIVO: $<10,33 \mathrm{fl}$

La estadística descriptiva permitió obtener el número de casos y su porcentaje para las variables cualitativas, mientras que las variables cuantitativas se han descrito mediante la media aritmética y la desviación típica, en el caso de variables cuantitativas asimétricas se utilizó la mediana, además de valores mínimo y máximo. Para la relación entre variables cuantitativas y cualitativas, se ha procedido a la descripción bivariante (medias y desviaciones para cada grupo) y la inferencia mediante un análisis de la varianza.

Para relacionar dos variables cualitativas, la descripción bivariante se estableció mediante frecuencias relativas presentadas en tablas de contingencia, a las que se les aplicó el test de chi cuadrado o test exacto de Fischer. La evaluación del rendimiento diagnóstico y pronóstico de las variables electrocardiográficas, puntaje de riesgo y biomarcadores (troponina, glicemia y volumen plaquetario medio) se ha evaluado mediante las medidas de validez: sensibilidad y especificidad, valor predictivo positivo y valor predictivo negativo, razón de verosimilitud positiva y razón de verosimilitud negativa. Para el análisis estadístico correspondiente, se ha calculado el intervalo de confianza (IC) del 95\%.

Además, cabe señalar que se ha empleado un análisis a través de las gráficas de curva ROC (Receiver Operator Curve o Curva Operante Receptor), con la finalidad de integrar la sensibilidad y especificidad, y establecer el mejor punto de cohorte en el cual se alcance la mejor sensibilidad (mayor detección de casos) con el menor número de falsos positivos. La significación de dichas curvas se ha evaluado a partir del cálculo del IC del área bajo la curva, interpretando como significativo aquel valor del área cuyo límite inferior fue superior al 0,5 . Se ha utilizado un modelo de regresión logística binaria. El software estadístico utilizado fue el SPSS (V20.0). La aproximación utilizada en todos los análisis ha sido bilateral y el nivel de significación del 5\% $(\alpha=0,05)$. 


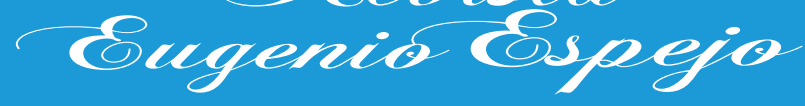

FACULTAD DE CIENCIAS DE LA SALUD

\section{FLUJOGRAMA DE SELECCIÓN DE PARTICIPANTES}

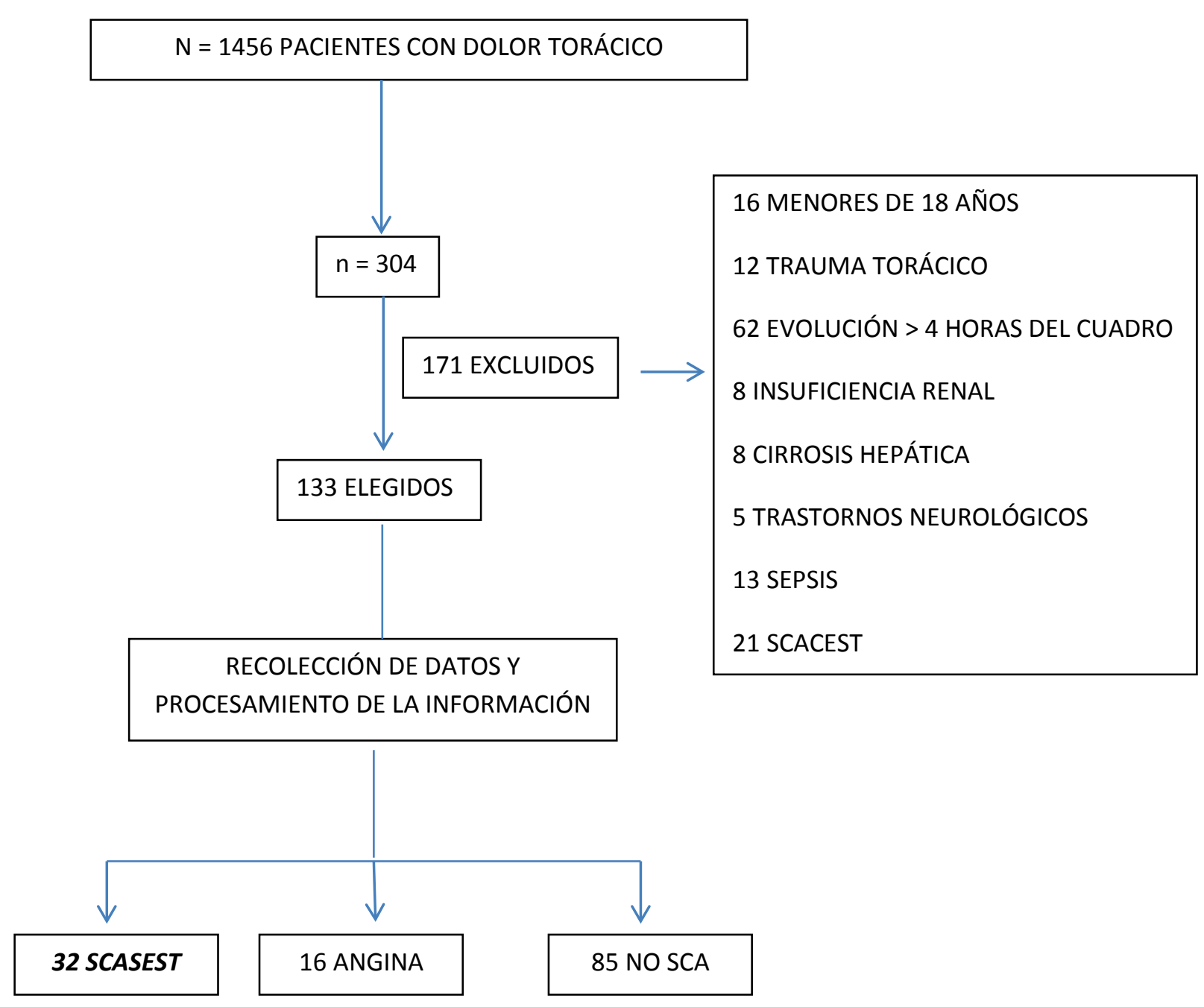

\section{RESULTADOS}

De los pacientes escogidos para el estudio (133), 77 (57,9\%) fueron hombres y 56 (42,1\%) mujeres, la media de edad en ambos grupos fue de 55,6 años con una desviación típica de 12,8 años con un mínimo de 23 años y un máximo 87 años (figura 1). Dentro de este mismo grupo, $32(24,1 \%)$ presentaron síndrome coronario agudo tipo infarto de miocardio sin elevación del segmento ST, siendo más frecuente en hombres 16 (12\%) angina inestable y 85 $(63,9 \%)$ no presentaban criterios para síndrome coronario agudo (causas extra cardíacas no contempladas dentro de los criterios de exclusión) (tabla 1). 
Tabla 1. Frecuencia de la población según diagnóstico y sexo.

\begin{tabular}{|l|r|r|c|}
\hline \multirow{2}{*}{ DIAGNÓSTICO } & \multicolumn{2}{|c|}{ SEXO } & \multirow{2}{*}{ TOTAL } \\
\cline { 2 - 3 } & MASCULINO & FEMENINO & \\
\cline { 2 - 3 } & No. & No. & No. \\
\hline IAMSEST & 27 & 5 & 32 \\
\hline ANGINA & 6 & 10 & 16 \\
\cline { 1 - 3 } NO SCA & 44 & 41 & 85 \\
\hline TOTAL & 77 & 56 & 133 \\
\hline
\end{tabular}

Abreviación. SCA: síndrome coronario agudo, IAMSEST: infarto agudo de miocardio sin elevación del segmento ST.

Se evidenció que el síndrome coronario agudo sin elevación del segmento ST fue más frecuente en hombres que en mujeres ( $84,4 \%$ vs $16 \%$ respectivamente). De la población estudiada, 97 casos $(72,9 \%)$ presentaron un electrocardiograma normal, 28 (21,1\%) inversión profunda y simétrica de la onda $\mathrm{T}$ en derivaciones precordiales y $8(6 \%)$ una depresión del segmento $\mathrm{ST} \geq 0,5 \mathrm{~mm}(0,05 \mathrm{mV})$ en 2 o más derivaciones contiguas. De igual manera al analizar la variable del puntaje de riesgo TIMI, se evidenció que 97 pacientes (72,9\%) estaban en categoría de bajo riesgo, $33(24,8 \%)$ en riesgo intermedio y $3(2,3 \%)$ en alto riesgo.

$\mathrm{Al}$ analizar las variables biológicas lo resultados fueron que la media de glicemia en la población de estudio fue de 123,29 mg/dl con una desviación típica de 19,77 mg/dl; el volumen plaquetario medio tuvo una media de 9,45 fl con una desviación de 0,95 y la troponina con una media de 88,71 ng/L y una desviación de 355,23 ng/L. (tabla 2)

Tabla 2. Descriptivos de los biomarcadores en el diagnóstico de síndrome coronario agudo sin elevación del ST

\begin{tabular}{|l|c|c|c|}
\hline \multicolumn{1}{|c|}{ MEDIDAS } & $\begin{array}{c}\text { GLUCOS } \\
\text { A (mg/dl) }\end{array}$ & $\begin{array}{c}\text { TROPONINA } \\
\text { (ng/L) }\end{array}$ & $\begin{array}{c}\text { VOLUMEN } \\
\text { PLAQUETARIO } \\
\text { MEDIO (fl) }\end{array}$ \\
\hline Media & 123,29 & 88,71 & 9,46 \\
\hline Desv. típ. & 19,78 & 355,24 &, 96 \\
\hline Mínimo & 87,00 & 2,15 & 7,16 \\
\hline Máximo & 176,00 & 3575,00 & 11,78 \\
\hline
\end{tabular}


Se procesaron datos relativos a los niveles de biomarcadores de forma individualizada para cada categoría de diagnóstico: infarto sin elevación del ST, angina inestable y no síndrome coronario agudo (tabla 3 ).

Tabla 3. Comparación de medias entre los diferentes biomarcadores, según categoría de diagnóstico

\begin{tabular}{|c|c|c|c|c|c|c|}
\hline \multicolumn{7}{|c|}{ GLUCOSA } \\
\hline DIAGNÓSTICO & Media & $\mathrm{N}$ & Desv. típ. & Mínimo & Máximo & IC 95\% \\
\hline IAMSEST & 145,37 & 32 & 9,44 & 132,00 & 176,00 & $141,97-148,78$ \\
\hline ANGINA & 126,00 & 16 & 16,30 & 103,00 & 154,00 & $117,32-134,68$ \\
\hline NO SCA & 114,47 & 85 & 16,39 & 87,00 & 156,00 & $110,94-118,00$ \\
\hline Total & 123,29 & $\begin{array}{r}13 \\
3 \\
\end{array}$ & 19,78 & 87,00 & 176,00 & \\
\hline \multicolumn{7}{|c|}{ VOLUMEN PLAQUETARIO MEDIO } \\
\hline DIAGNÓSTICO & Media & $\mathrm{N}$ & Desv. típ. & Mínimo & Máximo & IC 95\% \\
\hline IAMSEST & 10,42 & 32 & 0,65 & 8,45 & 11,78 & $10,19-10,65$ \\
\hline ANGINA & 9,77 & 16 & 0,79 & 8,10 & 10,65 & $9,35-10,19$ \\
\hline NO SCA & 9,03 & 85 & 0,79 & 7,16 & 10,56 & $8,87-9,20$ \\
\hline Total & 9,46 & 133 & 0,96 & 7,16 & 11,78 & \\
\hline \multicolumn{7}{|c|}{ TROPONINA } \\
\hline DIAGNÓSTICO & Media & $\mathrm{N}$ & Desv. típ. & Mínimo & Máximo & IC 95\% \\
\hline IAMSEST & 345,34 & 32 & 669,18 & 14,70 & 3575,00 & $104,07-586,60$ \\
\hline ANGINA & 8,39 & 16 & 3,36 & 3,10 & 13,30 & $6,60-10,18$ \\
\hline NO SCA & 7,22 & 85 & 2,74 & 2,15 & 13,70 & $6,63-7,81$ \\
\hline Total & 88,71 & 133 & 355,24 & 2,15 & 3575,00 & \\
\hline
\end{tabular}

La prevalencia de hiperglicemia ( $>140 \mathrm{mg} / \mathrm{dl}$ ) en el grupo de pacientes con infarto agudo de miocardio sin elevación del ST fue del 69,69\% y cuando se calculó la razón de prevalencia ésta fue del 7,66. Por otra parte, la prevalencia de pacientes con un volumen plaquetario medio mayor o igual a 10,33 fl fue del 78,78\% con una razón de prevalencia de 13 .

\section{Rendimiento diagnóstico}

\section{Volumen plaquetario medio (VPM)}

El análisis del rendimiento diagnóstico de las pruebas, estableció en primera instancia al evaluar la curva ROC que el mejor punto de cohorte para la población de estudio en lo que respecta a volumen plaquetario medio fue $\geq 10,33 \mathrm{fl}$ (AUC: 0,91; IC 95\%: 0,841-0,979; $\mathrm{p}<0,05)$. La significancia de la curva establece que posee una excelente capacidad predictiva para el diagnóstico de infarto agudo de miocardio sin elevación del ST. (figura 1) 
Figura 1. Curva ROC del volumen plaquetario medio para el diagnóstico de infarto agudo de miocardio sin elevación del ST.

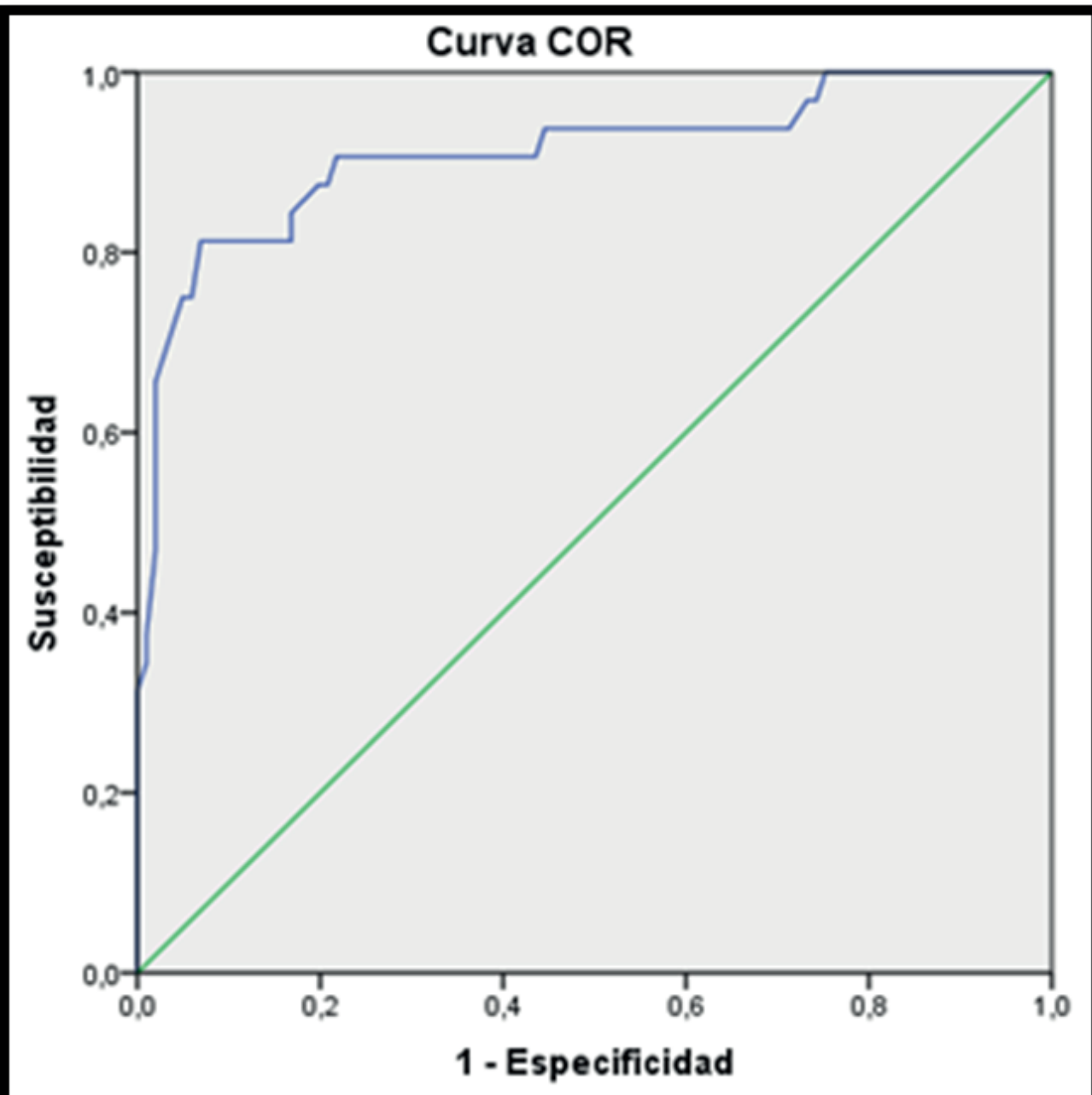

Área bajo la curva

Variables resultado de contraste: VPM

\begin{tabular}{|r|r|r|r|r|}
\hline Area & Error tip." & Sig. asintítica" & \multicolumn{2}{|c|}{$\begin{array}{l}\text { In tervalo de confianza asintótco al } \\
95 \%\end{array}$} \\
\cline { 4 - 5 } & & & Limite inferior & Limite superior \\
\cline { 4 - 5 } & & .000 & .841 & .979 \\
\hline
\end{tabular}

Al colocar los resultados obtenidos en la tabla de contingencia se determinó una sensibilidad del $81,2 \%$, especificidad de $93,1 \%$, porcentaje de falsos positivos $6,9 \%$, porcentaje de falsos negativos $18,8 \%$, valor predictivo positivo (VPP) $78,8 \%$, valor predictivo negativo (VPN) $94 \%$, razón de verosimilitud positivo $(\mathrm{RV}+)$ 11,723 y razón de verosimilitud negativo (RV-) 0,201 . 


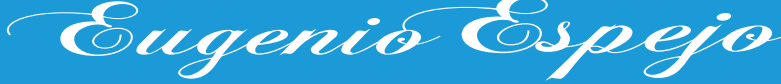

\section{Hiperglicemia (HG)}

El análisis estadístico estableció que el mejor punto de cohorte para el diagnóstico de infarto agudo de miocardio sin elevación del ST, en lo que respecta a la glicemia de la población en estudio, fue un valor superior a $140 \mathrm{mg} / \mathrm{dl}$ (AUC: 0,923; IC 95\%: 0,879-0,967; $\mathrm{p}<0,05$ ); permitiendo determinar según el área bajo la curva que la hiperglicemia posee una excelente capacidad predictiva para el diagnóstico de infarto agudo de miocardio sin elevación del segmento ST (figura 2)

Figura 2. Curva ROC de hiperglicemia para el diagnóstico de infarto agudo de miocardio sin elevación del ST.

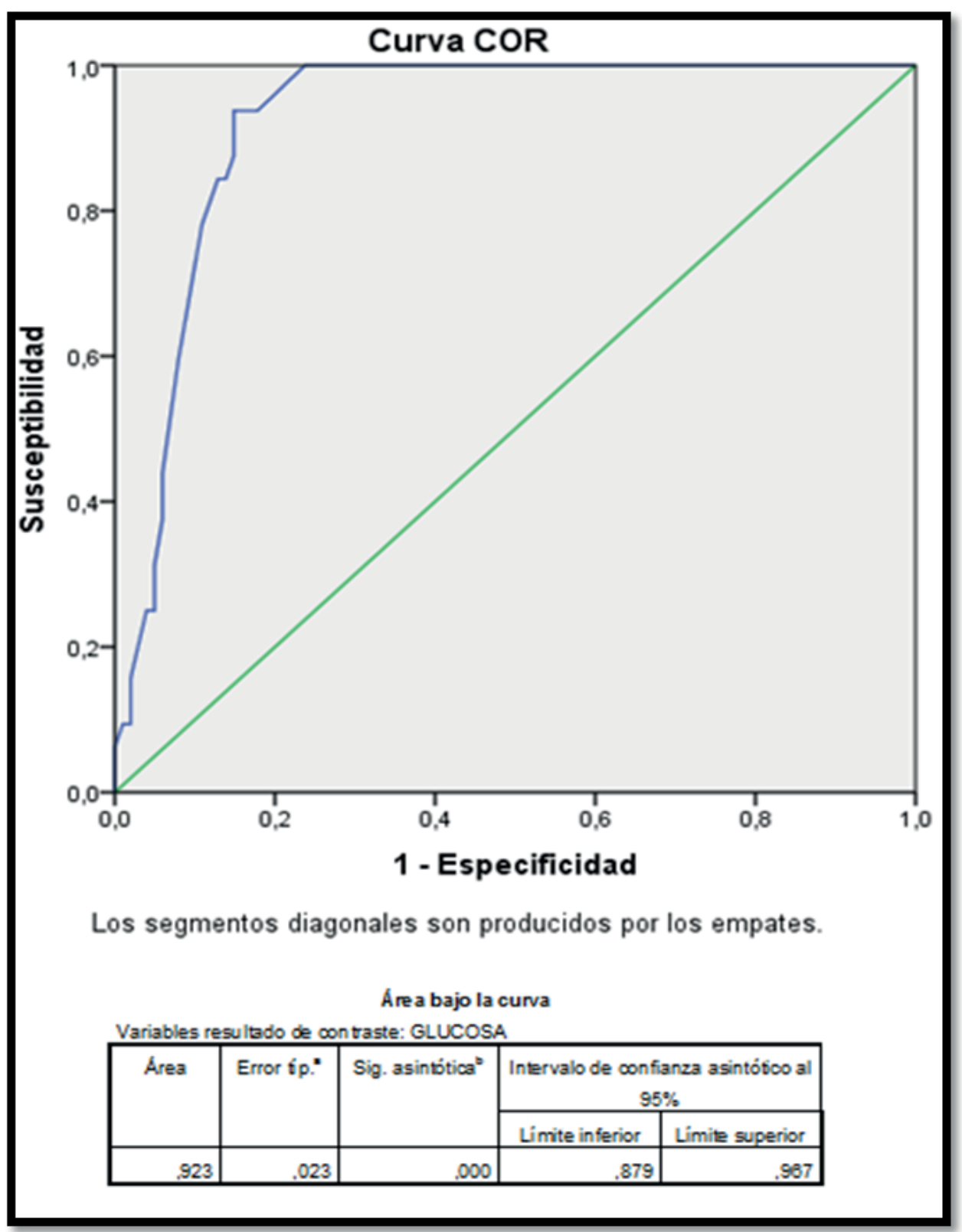


El análisis estadístico determinó que la hiperglucemia (>140 mg/dl) en el diagnóstico temprano del infarto agudo de miocardio sin elevación del segmento ST tiene una sensibilidad del 71,9\%, especificidad de 90,1\%, porcentaje de falsos positivos 9,9\%, porcentaje de falsos negativos $28,1 \%$, valor predictivo positivo (VPP) $69,7 \%$, valor predictivo negativo (VPN) 91\%, razón de verosimilitud positivo (RV+) 7,259 y razón de verosimilitud negativo (RV-) 0,312 .

\section{Comparación de modelos diagnósticos para el infarto agudo de miocardio sin elevación del segmento $\mathrm{ST}$}

Con la finalidad de establecer el modelo más idóneo para el diagnóstico del infarto agudo de miocardio sin elevación del segmento ST, se realizó combinaciones de parámetros clínicos, electrocardiográficos, puntaje de riesgo y biomarcadores. Los resultados obtenidos establecieron que el modelo 1 conformado por dolor torácico + cambios electrocardiográficos + puntaje TIMI + troponina T ultrasensible presenta la mejor significancia estadística, evidenciado por un área bajo la curva AUC de 0,797, IC 95\%: 0,688- 0,906, p <0,05.

Además, se estableció que la adición de la hiperglicemia y el volumen plaquetario medio (modelo 2) al modelo convencional (modelo 1) de diagnóstico no aporta un beneficio significativo. Sin embargo, cuando se utilizó un tercer modelo conformado por dolor torácico + cambios electrocardiográficos + puntaje TIMI + hiperglicemia + volumen plaquetario medio, su rendimiento diagnóstico a la admisión en pacientes con sospecha de síndrome coronario agudo tipo infarto de miocardio sin elevación del ST, fue muy comparable (AUC 0,761, IC $95 \%$ 0,647-0,874, $\mathrm{P}<0,05$ ) con el modelo convencional que utiliza entre sus variables a la troponina $\mathrm{T}$ ultrasensible. Un cuarto modelo conformado por dolor torácico + cambios electrocardiográficos + puntaje TIMI fue analizado presentando una AUC de 0,737, IC 95\% $0,625-0,849, \mathrm{p}<0,05$. (figura 3). 
Figura 3. Comparación de Curvas ROC de los diferentes modelos para el diagnóstico de infarto agudo de miocardio sin elevación del segmento ST.

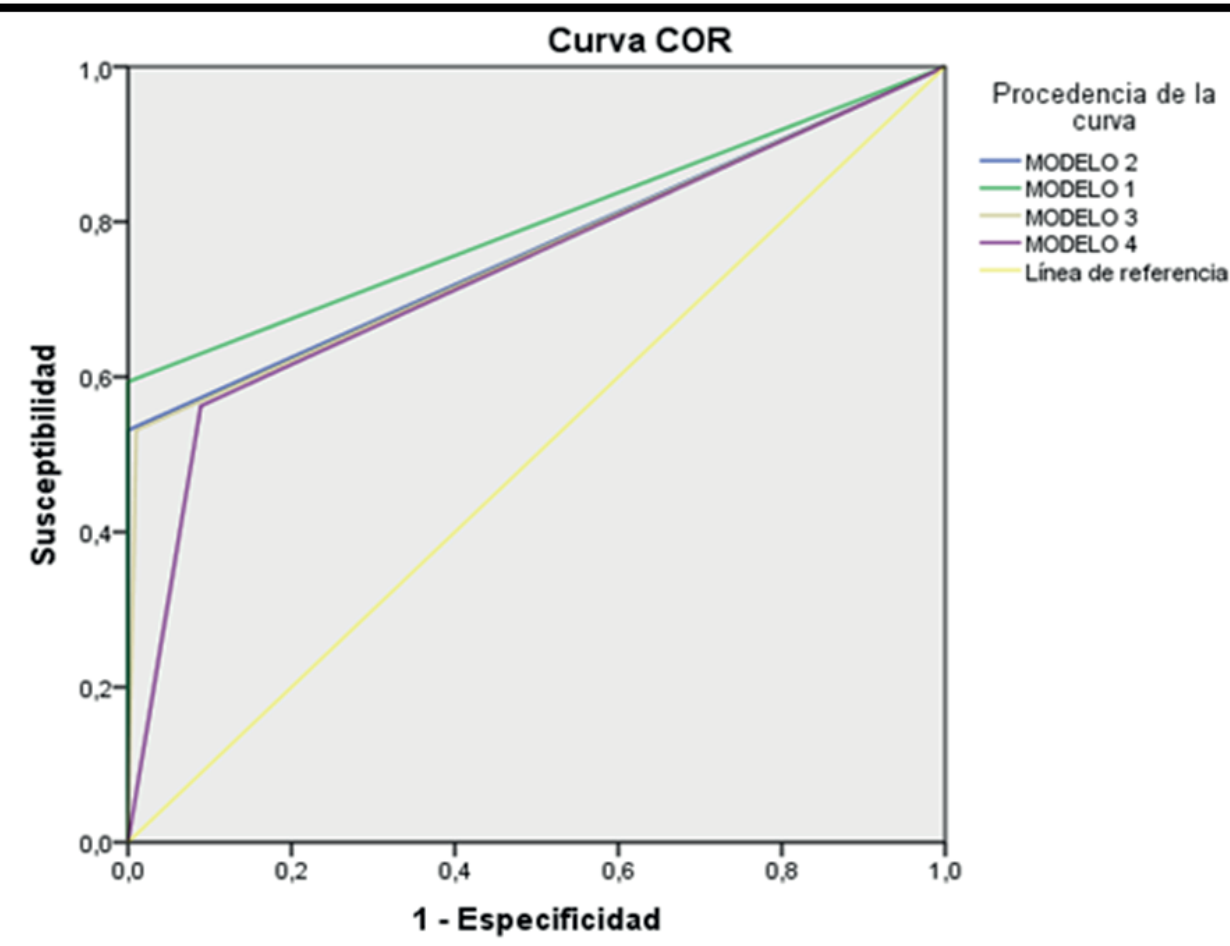

Los segmentos diagonales son producidos por los empates.

\begin{tabular}{|c|c|c|c|c|c|}
\hline \multicolumn{6}{|c|}{ Área bajo la curva (A UC) } \\
\hline \multirow{2}{*}{$\begin{array}{l}\text { Variables resultado de } \\
\text { contraste }\end{array}$} & \multirow{2}{*}{$\begin{array}{l}\text { Area } \\
\text { (AUC) }\end{array}$} & \multirow[t]{2}{*}{ Error tip. } & \multirow{2}{*}{$\begin{array}{c}\text { Sig. } \\
\text { Asintótcas }\end{array}$} & \multicolumn{2}{|c|}{ Intervalo de confianza asintótco al 95\% } \\
\hline & & & & Limite interior & Limite superior \\
\hline $\begin{array}{l}\text { MODELO } 1 \\
\text { (DT + ECG + TIMI + } \\
\text { Troponins) }\end{array}$ & .797 & .056 & .000 & .688 & .908 \\
\hline $\begin{array}{l}\text { MODELO } 2 \\
\text { (DT + ECG + TIMI + } \\
\text { Troponina + HG + VPM) }\end{array}$ & .786 & .058 & .000 & .652 & .879 \\
\hline $\begin{array}{l}\text { MODELO } 3 \\
\text { (DT + ECG + TIMI + HG } \\
+ \text { VPM) }\end{array}$ & .761 & 058 & .000 & .647 & .874 \\
\hline $\begin{array}{l}\text { MODELO } 4 \\
\text { (DT + ECG + TIMD) }\end{array}$ & .737 & .057 & .000 & .625 & .849 \\
\hline
\end{tabular}

Abreviación: DT: dolor torácico, ECG: electrocardiograma, TMI: puntaje de riesgo TMI,

HG: hiperglicemia, VPM: volumen plaquetario medio.

La mejor significancia estadística fue evidenciada con el modelo 1, principalmente en lo que respecta a confirmación de diagnóstico; no obstante, su valor predictivo negativo superior al de los otros modelos, aunque de forma discreta, lo convierte en una herramienta útil en la práctica clínica para descartar la posibilidad de un síndrome coronario agudo en pacientes que acuden al Departamento de Emergencia por dolor torácico (tabla 4). 
Tabla 4. Rendimiento diagnóstico de la combinación de parámetros clínicos, electrocardiográficos, puntaje de riesgo TIMI y biomarcadores en el infarto agudo de miocardio sin elevación del segmento ST.

\begin{tabular}{lrrrrrrrr}
\hline & SEN \% & ESP \% & $\begin{array}{r}\text { VPP } \\
\text { \% }\end{array}$ & $\begin{array}{r}\text { VPN } \\
\text { \% }\end{array}$ & RV+ & $\begin{array}{r}\text { Ppost (+) } \\
\text { \% }\end{array}$ & RV- & $\begin{array}{r}\text { Ppost (-) } \\
\text { \% }\end{array}$ \\
\hline MODELO 1 & 66,7 & 100 & 100 & 88,6 & 66,37 & 100 & 0,33 & 20,1 \\
\hline MODELO 2 & 53,1 & 100 & 100 & 87,0 & 53,63 & 100 & 0,47 & 26,1 \\
\hline MODELO 3 & 53,1 & 99,0 & 94,4 & 87,0 & 53,66 & 97,6 & 0,47 & 26,3 \\
\hline MODELO 4 & 56,2 & 91,1 & 66,7 & 86,8 & 6,31 & 82,6 & 0,48 & 26,6 \\
\hline
\end{tabular}

Abreviación: SEN: sensibilidad, ESP: especificidad, F.P.: falsos positivos, F.N.: falsos negativos, VPP: valor predictivo positivo, VPN: valor predictivo negativo, $\mathrm{RV+}$ +: razón de verosimilitud positivo, RV-: razón de verosimilitud negativo, Ppost: probabilidad postest.

\section{DISCUSIÓN}

El diagnóstico de un paciente con síndrome coronario agudo se basa principalmente en cuatro pilares: $^{(5-7)}$

1. El cuadro clínico representado por el dolor torácico, no siempre de características típicas y ausente en muchos casos.

2. Factores de riesgo cardiovascular que permiten categorizar a los pacientes y evaluar la probabilidad de un evento isquémico.

3. Hallazgos electrocardiográficos cuya utilidad clínica dependerá en primer lugar del grado de capacitación del profesional que lo interprete y en segundo lugar de su presencia al momento del evento, tal es el caso del síndrome coronario agudo tipo infarto agudo de miocardio sin elevación del ST donde en gran proporción estos hallazgos están ausentes.

4. Biomarcadores cardiacos, los mismos que no siempre están disponibles en una unidad de salud y si lo están sus resultados no son inmediatos o carecen de niveles adecuados de sensibilidad y especificidad en etapas tempranas del evento (las primeras 4-6 horas).

Desafortunadamente, ningún marcador sérico usado es "ideal" para el diagnóstico precoz de síndrome coronario agudo, al carecer de alguna de las tres características fundamentales: elevada sensibilidad, elevada especificidad y pronta elevación, de ser posible dentro de las 6 primeras horas, que es precisamente la ventana temporal de máximo interés para los médicos de Urgencias para la toma de decisiones diagnóstico-terapéuticas. Las limitaciones de todos los procedimientos diagnósticos llevaron a la búsqueda de marcadores de daño miocárdico, cuya elevación se produzca en fases más tempranas de la isquemia. ${ }^{(4)}$ Se estima que son dados de alta erróneamente entre el 5 al 10\% de los casos diagnosticados de infarto agudo de miocardio y el $2 \%$ de las anginas inestables. ${ }^{(8)}$ 
Nosotros encontramos que tanto el volumen plaquetario medio en un punto de cohorte superior a 10,33 fl como la hiperglicemia a la admisión $>140 \mathrm{mg} / \mathrm{dl}$ son predictores de un infarto agudo de miocardio sin elevación del ST, dentro de las primeras 4 horas de inicio del cuadro clínico. ${ }^{(6)}$ Un estudio evidenció que pacientes adultos chinos que acudieron al servicio de Emergencia por dolor torácico, el volumen plaquetario medio fue un predictor independiente de síndrome coronario agudo con un punto de cohorte de $10,35 \mathrm{fl}^{(9-11)}$

En los pacientes con IAM se ha demostrado un aumento del volumen plaquetario medio junto a un desplazamiento hacia la derecha de la curva de distribución del mismo, en comparación con los pacientes ingresados por dolor torácico, pero sin evidencia de infarto de miocardio. $^{(12-14)}$

De igual manera, Charpentier et al en un estudio prospectivo de 11 meses de duración, estableció que un valor de glicemia superior a $140 \mathrm{mg} / \mathrm{dl}$ está asociado con síndrome coronario agudo, tipo infarto de miocardio sin elevación del ST. ${ }^{(13)}$ La intolerancia a los hidratos de carbono está asociada a síndrome coronario agudo, según un estudio de cohorte con 140 pacientes admitidos por síndrome coronario agudo. Una glucosa en ayunas $\geq$ de $100 \mathrm{mg} / \mathrm{dl} \mathrm{o}$ a la admisión $\geq$ de $140 \mathrm{mg} / \mathrm{dl}$ tuvo una sensibilidad del $89.5 \%$ para detectar diabetes y un valor predictivo positivo del $43.6 \%{ }^{(15)}$

A la hiperglicemia, también se la realciona con el pronóstico y la mortalidad de los pacientes con síndrome coronario agudo; así, una revisión sistemática de 15 estudios entre los años 1966 a 1998 demostró un riesgo relativo de mortalidad hospitalaria 3.9 veces mayor en pacientes no diabéticos con IAM cuando tuvieron una glicemia de ingreso $\geq 110 \mathrm{mg} / \mathrm{dl} \mathrm{com}$ parado con los que ingresaron normoglucémicos, mientras que para pacientes diabéticos con una glicemia de ingreso $\geq$ de $180 \mathrm{mg} / \mathrm{dl}$, el riesgo relativo de muerte fue 1.7 veces mayor que en los diabéticos normoglucémicos al ingreso. ${ }^{(16-18)}$

Al comparar diferentes modelos de diagnóstico de síndrome coronario agudo, se estableció que el modelo tradicional (dolor torácico, puntaje de riesgo TIMI, hallazgos electrocardiográficos y niveles de troponina) tuvo una sensibilidad del $66,7 \%$, especificidad del $100 \%$, VPP de 100\%, VPN 88,6\%, RV+ 66,37 y RV- de 0,33, destacando su utilidad en la práctica clínica como una herramienta confirmatoria más que excluyente. En contraste con otros protocolos que incluyen hallazgos electrocardiográficos, TIMI y niveles de troponina donde por su destacada sensibilidad con el 99,7\% y un VPN 99,7\%, se convierte en un método útil en la exclusión de la enfermedad. ${ }^{(19)}$

La adición del volumen plaquetario medio y la hiperglucemia al modelo clásico de diagnóstico no mejora de forma significativa el diagnóstico de infarto de miocardio, por lo que su rol 
principal está determinado cuando se analiza de forma independiente. Lo mencionado anteriormente es corroborado por Charpienter et al cuando comparó un primer modelo de diagnóstico frecuente versus el mismo modelo con adición de la hiperglucemia (AUC 0.82, 95\% CI 0.78 a 0.85 Vs AUC adicionado hiperglucemia: $0.82,95 \%$ CI 0.79 a 0.85$){ }^{(13)}$

La prevalencia de hiperglucemia ( $>140 \mathrm{mg} / \mathrm{dl})$ en pacientes con infarto agudo de miocardio sin elevación del ST en nuestro estudio fue cercana al 70\%, en comparación con el descrito por Deedwania et al que oscila entre el 51 al $58 \%{ }^{(20)}$ y con Zarich et al del 70\%.(7,18,21)

En la actualidad existen más estudios que abordan el vínculo entre la hiperglucemia, el volumen plaquetario medio y el pronóstico a corto plazo en pacientes con infarto agudo de miocardio; sin embargo, no hay suficiente información referente al rol de éstos biomarcadores en el diagnóstico inicial.

\section{Limitaciones}

Al tratarse de un estudio con análisis post facto, algunas de las variables independientes no pudieron ser controladas de forma directa, principalmente aspectos técnicos en la fase pre analítica y de procesamiento de las muestras (tiempo, tipo de anticoagulante). Por otra parte, a pesar de establecer criterios definidos de inclusión y exclusión, existen factores no controlables (etnia, edad, enfermedades previas, uso de medicamentos, hábitos) que pudieron incidir en los valores del volumen plaquetario medio y la glicemia al momento de la admisión. La estrecha selección de los pacientes permitió exonerar a un grupo importante de la población, por lo que los resultados no pueden ser extrapolados de forma amplia.

\section{CONCLUSIÓN}

La hiperglucemia ( $>140 \mathrm{mg} / \mathrm{dl})$ y el volumen plaquetario medio ( $\geq 10,33 \mathrm{fl})$ son altamente prevalentes en pacientes con infarto agudo de miocardio sin elevación del ST, y su determinación a la admisión, en el contexto clínico y electrocardiográfico, tiene una buena capacidad predictiva de enfermedad isquémica coronaria aguda.

\section{Agradecimientos}

Al personal de salud del Hospital Carlos Andrade Marín de la ciudad de Quito, por permitir la realización del presente trabajo de investigación, facilitándonos toda la información pertinente; así como también, el acceso a las diferentes bases de datos intrahospitalarias.

\section{Conflictos de intereses}

Ninguno 


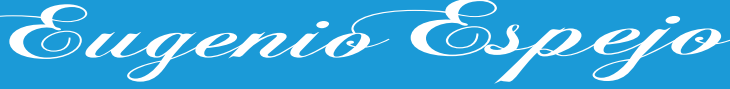

FACULTAD DE CIENCIAS DE LA SALUD

\section{Declaración de contribución}

HF Ortega, trabajó en la concepción, análisis estadístico, tabulación de datos y redacción final. JC Piedra, trabajó en la recolección y organización de los datos.

\section{REFERENCIAS BIBLIOGRÁFICAS}

1. Alquezar BLB. Clinical Scales: Tools for the Diagnosis of Unstable Angina. Revista Clínica Española. [Internet]. 2018 [citado 17 Jun 2018]; 218(1): 1-2. Disponible en:

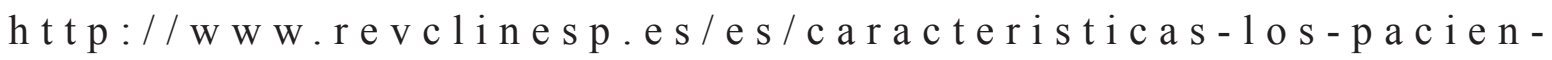
tes-con-derrame/articulo/S0014256517302618/.

2. Narvaez A. Infarto Agudo de Miocardio. SCACEST. Servicio de Cardiologia [Internet]. 2017 diciembre [citado 22 Jun 2018]; 70(12): 1039 - 1045. Disponible en: http://appswl.else-

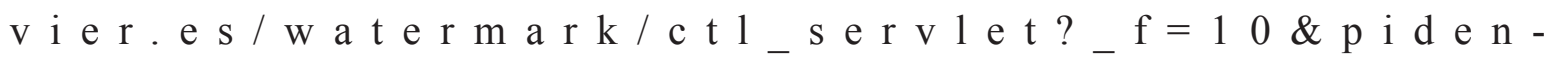
$\mathrm{t} \_$articulo $=90461837 \&$ pident_usuario $=0 \&$ pcontactid $=\&$ pident_revista $=25 \&$ ty $=101 \&$ accion $=$ L\&origen $=$ cardio \&we $=$ www.revespcardiol.org\&lan $=$ es \& fichero $=25 \mathrm{v} 70 \mathrm{n} 12 \mathrm{a} 90461837 \mathrm{p}$ df001.pdf\&anuncioPdf=ERROR_publi_pdf.

3. OPS. Representación Ecuador. Quito: Comunigraf S.A.; 2014.

4. García L, Castrillo R, Valle A, Suárez A, Soler W, Martín F, et al. Diagnóstico Precoz del Síndrome Coronario Agudo en los Servicios de Urgencias. Papel de la h-FABP. Emergencias. 2004 Feb; 16: 233-238.

5. Steg G, James S, Atar D, Badano L, et al. Guía de Práctica Clínica de la ESC para el manejo del Infarto Agudo de Miocardio en pacientes con elevación del segmento ST. Revista Española de Cardiologia [Internet]. 2013 enero [citado 22 Junio 2018]; 66(1): 1 - 46. Disponible en: http://www.revespcardiol.org/es/guia-practica-clinica-esc-el/articulo/90180910/.

6. Gutiérrez A, et al. Volumen Plaquetario Medio: El tamaño si importa. Med int mex [Internet]. 2013 mayo [citado 22 Junio 2018]; 29(3): 307-310. Disponible en: http://www.medigraphic.com/pdfs/medintmex/mim-2013/mim133k.pdf.

7. García De Gea, et al. Markers for Early Detection of Alterations in Carbohydrate Metabolism after acute Myocardial Infarction. Medicina Intensiva [Internet]. 2013 abril [citado 22 Junio 2018]; 38(1): 83-91. Disponible en:https://europepmc.org/abstract/med/23623422.

8. Pope JH, Aufderheide TP, Ruthazer R, Woolard RH, Feldman JA, Beshansk J, et al. Missed Diagnoses of Acute Cardiac Ischemia in the Emergency Department. N Engl J Med [Internet]. 2000 [citado 05 mayo 2017]; 342: 1163-70. Disponible en: https://www.nejm.org/doi/pdf/10.1056/NEJM200004203421603.

9. Hsin, Chu, Wei-Lung Chen, Chien-Cheng Huang, Hsin-Yu Chang, Hung-Yi Kuo, Chorng-Ming Gau, et al. Diagnostic Performance of Mean Platelet Volume for Patients with Acute 
Coronary Syndrome visiting an Emergency Department with Acute Chest Pain: The Chinese Scenario. EmergMed J [Internet]. 2011 [citado 05 mayo 2017]; 28: 569-574. Disponible en: https://emj.bmj.com/content/28/7/569.long.

10. Gómez D, Sanchez G, Perez M, Garcia R, Jaramillo P. et al, Hyperglycaemia is associated with worse outcomes in Latin-American individuals with Acute Myocardial Infarction. Clínica e Investigación en Arteriosclerosis [Internet]. 2015 enero [citado 22 Junio 2018]; 28(1): 1-10. Disponible en: http://www.elsevier.es/es-revista-clinica-e-investigacion-arteriosclerosis-15-pdf-S0214916815001291-S300.

11. Erne P, Wardle J, Sanders K, Lewis SM, Maseri A. Mean Platelet Volume and Size Distribution and their Sensitivity to agonists in patients with Coronary Artery Disease and Congestive Heart Failure. Throm Haemostas [Internet]. 1988 [citado 15 Mayo 2017]; 59: 259-263. Disponible en: https://www.ncbi.nlm.nih.gov/pubmed/3388297.

12. Martin JF, Plum J, Kilbey RS, Kishk YT. Changes in Volume and Density of Platelets in Myocardial Infarction. Br Med J [Internet]. 1983 [citado 15 Mayo 2017]; 287:456-459. Disponible en: https://www.ncbi.nlm.nih.gov/pmc/articles/PMC1548755/pdf/bmjcred00566-0018.pdf.

13. Charpentier S, Cournot M, Lauque D, Girardi C, Bounes V, Elbaz M, et al. Usefulness of initial glucose level to improve Acute Coronary Syndrome Diagnosis in the Emergency Department. EmergMed J [Internet]. 2011 [citado 15 Mayo 2017]; 28:564-568. Disponible en: https://emj.bmj.com/content/28/7/564.long.

14. Ruesen P. Large Platelets continue to circulate in an activated state after Myocardial Infarction. Eur J Clin Invest [Internet]. 1994 [citado 15 Mayo 2017]; 24: 243-247. Disponible en: https://onlinelibrary.wiley.com/doi/pdf/10.1111/j.1365-2362.1994.tb01081.x.

15. Okosieme O, Peter R, Usman M, Bolusani H, Suruliram P, George L, et al. Can admission and fasting glucose reliably identify undiagnosed diabetes in patients with acute coronary syndrome?. Diabetes Care [Internet]. 2008 [citado 15 Mayo 2017]; 31(10): 1955-9. Disponible en: https://www.ncbi.nlm.nih.gov/pmc/articles/PMC2551634/pdf/1955.pdf.

16. Capes S, Hunt D, Malmberg K, Gerstein H. Stress Hyperglycemia and Increased Risk of Death After Myocardial Infarction in patients with and without Diabetes: A Systematic Overview. Lancet [Internet]. 2000 [citado 10 Mayo 2017]; 35:773-8. Disponible en: https://www.thelancet.com/pdfs/journals/lancet/PIIS0140-6736(99)08415-9.pdf.

17. Trowbridge EA, Martin JF. The Platelet Volume Distribution: A Signature of the Prethrombotic State in Coronary Heart Disease? Thromb Haemostas [Internet]. 1987 [citado 15 Mayo 2017]; 58: 714-717. Disponible en: https://www.ncbi.nlm.nih.gov/pubme$\mathrm{d} / 3672422$.

18. Richard w. Nesto sei. Glycemic Control for Acute Myocardial Infarction in patients with and without Diabetes Mellitus Uptodate [Internet]. 2018 abril [citado 22 Junio 2018]; 58(14): 1 - 8. Disponible en: https://www.uptodate.com/contents/glycemic-control-for-acute-myocardial-infarction-in-patients-with-and-without-diabetes-mellitus. 
19. Than M, Cullen L, Aldous S, Parsonage W, Reid C, Greenslade J, et al. 2-Hour Accelerated Diagnostic Protocol to Assess Patients with Chest Pain Symptoms Using Contemporary Troponins as the Only Biomarker. The ADAPT Trial. JACC [Internet]. 2012 [citado 10 Mayo 2017]; 59(23): 2091-98. Disponible en: http://www.medpagetoday.com/upload/2012/5/29/2091.pdf.

20. Deedwania P, Kosiborod M, Barrett E, et al. Hyperglycemia and Acute Coronary Syndrome. A Scientific Statement from the American Heart Association Diabetes Committee of the Council of Nutrition, Physical Activity, and Metabolism. Circulation [Internet]. 2008 [citado 10 Mayo 2017]; 117: 1610-9. Disponible en: http://circ.ahajournals.org/content/117/12/1610.long.

21. Zarich SW, Nesto RW. Implications and Treatment of Acute Hyperglycemia in the Setting of Acute Myocardial Infarction. Circulation [Internet]. 2007 [citado 10 Mayo 2017]; 115: 436-9. Disponible en: http://circ.ahajournals.org/content/115/18/e436.long.

Recibido: 03 de julio de 2018

Aprobado: 25 de enero de 2019 